\title{
无穷维 Hamilton 算子广义本征函数系的完备性 及其在弹性力学中的应用
}

\author{
侯国林*，阿拉坦仓 \\ 内蒙古大学数学科学学院, 呼和浩特 010021 \\ E-mail: houguolin@163.com, alatanca@imu.edu.cn \\ 收稿日期: 2011-05-09；接受日期: 2011-11-30; * 通信作者 \\ 国家自然科学基金 (批准号: 10962004) 及内蒙古自然科学基金 (批准号: 20080404MS0104) 资助项目
}

摘要 本文利用无穷维 Hamilton 算子的结构特性, 得到由算子的基本本征函数和若当型本征函数构 成的广义本征函数系在 Cauchy 主值意义下完备的充分必要条件. 进而将结果应用于弹性力学中的板 弯曲问题. 相应结论为 Hamilton 体系下的分离变量法 (弹性力学求解新体系) 提供了理论保证.

关键词 Hamilton 系统 无穷维 Hamilton 算子 完备性 Cauchy 主值 板弯曲方程

MSC (2000) 主题分类 47A75, 74B10, 47A45

\section{1 引言}

数学物理中的许多偏微分方程 (组) 都可化为如下的无穷维 Hamilton 正则系统 ${ }^{[1-4]}$

$$
\frac{\partial u}{\partial t}=J^{-1} \frac{\delta \mathscr{H}}{\delta u},
$$

其中 $\mathscr{H}$ 为 Hamilton 泛函, $\delta / \delta u$ 为变分导数. 根据变分理论中势算子的 Vainberg 定理 ${ }^{[5]}$, 线性偏微 分方程 (组) 的 Hamilton 系统可等价地表示为

$$
\frac{\partial U}{\partial t}=H U
$$

这里 $U$ 为状态空间中的向量值函数, $H=\left[\begin{array}{cc}A & B \\ C & -A^{*}\end{array}\right]$ 就是无穷维 Hamilton 算子.

对于可转化为白伴算子本征值问题的偏微分方程, 传统的分离变量法是一种十分有效的求解方 法. 然而, 力学中的许多问题是非自伴的, 由于不能保证基底函数系的完备性, 传统的分离变量法就显 得无能为力. 为突破自伴性的限制去求解弹性力学问题, 钟万睤教授 ${ }^{[6]}$ 将 Hamilton 体系引入了弹性 力学, 建立了弹性力学求解新体系, 理性求解了许多应用力学问题 ${ }^{[7-10]}$. 求解新体系的本质是基于无 穷维 Hamilton 算子的分离变量法. Hamilton 算子一般是无界非自伴算子, 新体系方法是否可行, 需要 利用算子矩阵理论 ${ }^{[11]}$ 深入研究无穷维 Hamilton 算子的谱理论, 其中 Hamilton 算子本征函数系的完 备性是首要解决的问题.

最近, 我们证明了对各向同性电磁弹性固体问题采用 Hamilton 体系下的分离变量法是可行的 [12]. 后来, 经证明平面弹性 ${ }^{[13]}$ 及矩形中厚板问题 [14] 在简支边界条件下的相应 Hamilton 算子的本征函 数系在 Cauchy 主值意义下都是完备的. 文献 [15] 针对一类无穷维 Hamilton 算子, 利用内部算子的性 
质, 建立了整个算子的基本本征函数系在 Cauchy 主值意义下的完备性定理. 之后, 本文作者得到了斜 对角无穷维 Hamilton 算子的基本本征函数系在 Cauchy 意义下完备的充要条件 [16]. 文献 [15] 中的结 论可用于判别波动方程、热传导方程和 Laplace 方程等问题导出的 Hamilon 算子基本本征函数系的 完备性, 但结论成立的前提假设较强, 目前还不能用于判别来源于实际力学问题的 Hamilton 算子本征 函数系的完备性.

本文得到了一类无穷维 Hamilton 算子的广义本征函数系在 Cauchy 主值意义下的完备性定理, 并 将结果应用于弹性力学中的板弯曲方程. 广义本征函数系由基本本征函数和 Jordan 型本征函数组成. 我们的结果与力学学者的实践相一致, 即 Hamilton 算子的基本本征函数系不完备时, 要得到完备的本 征函数系应由该算子的 Jordan 型本征函数加以补充.

\section{2 预备知识和引理}

除非特别说明, 文中始终用 $X$ 表示 Hilbert 空间. $i \mathbb{R}$ 表示全体纯虚数构成的集合. $\mathcal{D}(T)$ 和 $\mathcal{R}(T)$ 表示线性算子 $T$ 的定义域和值域. $\sigma_{p}(T), \sigma_{r}(T)$ 和 $\sigma_{c}(T)$ 分别表示线性算子 $T$ 的点谱、剩余谱和连续 谱, 集合 $\sigma(T)=\sigma_{p}(T) \cup \sigma_{r}(T) \cup \sigma_{c}(T)$ 为 $T$ 的谱集, $\rho(T)=\mathbb{C} \backslash \sigma_{p}(T)$ 为 $T$ 的预解集. 为叙述简洁, 用 统一符号 $\langle\cdot, \cdot\rangle$ 表示相应 Hilbert 空间中的内积. 乘积空间 $X \times X$ 中的内积按通常定义. 若 $\lambda \in \mathbb{C}$, 我 们用 $\bar{\lambda}$ 表示 $\lambda$ 的复共轭. 称稠定闭线性算子 $T$ 是可逆的, 如果 $T$ 为双射且 $T^{-1}$ 为有界线性算子.

下面叙述一些定义.

定义 2.1 设 $H=\left[\begin{array}{cc}A & B \\ C & -A^{*}\end{array}\right]: \mathcal{D}(H) \subset X \times X \rightarrow X \times X$ 为稠定闭线性算子, 如果 $A$ 为稠定闭线 性算子, $B$ 和 $C$ 为自伴线性算子, 则称 $H$ 为无穷维 Hamilton 算子. 此时, 称下列发展方程

$$
\frac{\partial U(t, y)}{\partial t}=H U(t, y)
$$

为无穷维 Hamilton 系统.

定义 $2.2 X$ 中的函数系 $\left\{r_{n}\right\}_{n=-\infty}^{+\infty}$ 称为 Cauchy 主值意义下完备的, 如果对于任意的 $x \in X$ 都存在常数序列 $\left\{C_{ \pm n}\right\}_{n=1}^{+\infty}$ 满足

$$
x=\sum_{n=1}^{+\infty}\left(C_{n} r_{n}+C_{-n} r_{-n}\right)
$$

即

$$
\lim _{N \rightarrow+\infty}\left\|x-\sum_{n=1}^{N}\left(C_{n} r_{n}+C_{-n} r_{-n}\right)\right\|=0 .
$$

定义 2.3 设 $\lambda$ 是空间 $X$ 中线性算子 $T$ 的本征值. 如果存在非零元 $u^{0} \in \mathcal{D}(T)$ 使得 $T u^{0}=\lambda u^{0}$, 则 $u^{0}$ 称为 $T$ 的基本本征函数. 如果存在 $u^{1} \in \mathcal{D}(T)$ 使得

$$
T u^{1}=\lambda u^{1}+u^{0}, \quad u^{1} \neq 0,
$$

则 $u^{1}$ 被称为 $T$ 的 (一阶) Jordan 型本征函数. 一般地, 如果 $T$ 的 $k-1$ 阶 Jordan 型本征函数 $u^{k-1}$ 已 经定义, 那么它的 $k$ 阶 Jordan 型本征函数 $u^{k}$ 由下式定义

$$
T u^{k}=\lambda u^{k}+u^{k-1}, \quad u^{k} \neq 0 .
$$


注 2.1 由于实际力学问题出现的许多无穷维 Hamilton 算子其非零本征值对应的本征函数最 多出现一阶 Jordan 型, 如板弯曲问题 [7]、平面弹性问题 [17] 等. 下文仅就仅出现一阶 Jordan 型本征 函数情形进行讨论.

为了证明文中的主要结论, 先陈述一些引理.

引理 2.1 设 $Q_{i}^{0}$ 和 $Q_{i}^{1}$ 为无穷维 Hamilton 算子 $H$ 相应于本征值 $\lambda_{i}(i=1,2)$ 的基本本征函 数和 (一阶) Jordan 型本征函数, 若 $\lambda_{1}+\bar{\lambda}_{2} \neq 0$, 则下列辛正交性成立:

$$
\left\langle Q_{1}^{0}, J Q_{2}^{0}\right\rangle=\left\langle Q_{1}^{0}, J Q_{2}^{1}\right\rangle=\left\langle Q_{1}^{1}, J Q_{2}^{1}\right\rangle=0
$$

其中

$$
J=\left[\begin{array}{cc}
0 & I \\
-I & 0
\end{array}\right]
$$

下面始终设无穷维 Hamilton 算子 $H$ 满足如下两个假设: (i) $H$ 是可逆的且具有至多可数个本征 值; (ii) $B$ 是可逆的, $A$ 是从 $X$ 到 $X$ 的有界算子, 且 $B^{-1} A$ 为白伴算子.

由条件 (ii), 知

$$
\left(B^{-1} A\right)^{*}=A^{*} B^{-1}=B^{-1} A,
$$

这个关系式在后文起重要作用.

引理 2.2 在假设 (i)-(ii) 下, $H$ 具有如下谱性质:

(1) $\sigma(H), \sigma_{p}(H) \cup \sigma_{r}(H), \sigma_{c}(H)$ 和 $\rho(H)$ 分别关于虚轴对称. 当 $\sigma_{r}(H) \neq \emptyset$ 时, $\sigma_{r}(H)$ 不关于虚 轴对称;

(2) $\sigma_{p}(H), \sigma_{r}(H)$ 和 $\sigma_{c}(H)$ 分别关于坐标原点中心对称, 进而 $\sigma(H), \rho(H)$ 也分别具有同样性质;

(3) $\sigma_{p}(H)=\left\{\lambda \in \mathbb{C} \mid \lambda^{2} \in \sigma_{p}\left(A^{2}+B C\right)\right\}=\left\{\lambda \in \mathbb{C} \mid \lambda^{2} \in \sigma_{p}\left(A^{* 2}+C B\right)\right\}$;

(4) $\sigma_{r}(H)=\left\{\lambda \in \mathbb{C} \mid \lambda^{2} \notin \sigma_{p}\left(A^{2}+B C\right)\right.$ 且 $\left.\bar{\lambda}^{2} \in \sigma_{p}\left(A^{* 2}+C B\right)\right\}=\left\{\lambda \in \mathbb{C} \mid \lambda^{2} \notin \sigma_{p}\left(A^{* 2}+\right.\right.$ $C B)$ 且 $\left.\bar{\lambda}^{2} \in \sigma_{p}\left(A^{2}+B C\right)\right\}$;

(5) $\sigma_{c}(H)=\left\{\lambda \in \mathbb{C} \mid \lambda^{2} \notin \sigma_{p}\left(A^{2}+B C\right)\right.$ 且 $\left.\bar{\lambda}^{2} \notin \sigma_{p}\left(A^{* 2}+C B\right)\right\} \cap\left\{\lambda \in \mathbb{C} \mid \mathcal{R}\left(A^{* 2}+C B-\lambda^{2}\right) \neq\right.$ $X$ 或 $\left.\mathcal{R}\left(A^{2}+B C-\lambda^{2}\right) \neq X\right\}$.

证明 注意到 $J=-J^{*}=-J, J^{2}=\operatorname{diag}(I, I)$ 且 $A$ 是有界线性算子, 因此可得

$$
J H=\left[\begin{array}{cc}
0 & I \\
-I & 0
\end{array}\right]\left[\begin{array}{cc}
A & B \\
C & -A^{*}
\end{array}\right]=\left[\begin{array}{cc}
C & -A^{*} \\
-A & -B
\end{array}\right]=-H^{*} J
$$

故

$$
H^{*}=-J H J^{-1}
$$

即 $H$ 为辛自伴算子. 根据文献 [18] 中定理 3.6, 3.8, 3.14 和 3.15 知 (1) 成立.

若 $B^{-1} A$ 也为自伴算子, 则

$$
\left[\begin{array}{cc}
I & 0 \\
B^{-1} A & I
\end{array}\right]^{-1}=\left[\begin{array}{cc}
I & 0 \\
-B^{-1} A & I
\end{array}\right]
$$


且

$$
\left[\begin{array}{cc}
A & B \\
C & -A^{*}
\end{array}\right]=\left[\begin{array}{cc}
I & 0 \\
-B^{-1} A & I
\end{array}\right]\left[\begin{array}{cc}
0 & B \\
C+B^{-1} A^{2} & 0
\end{array}\right]\left[\begin{array}{cc}
I & 0 \\
B^{-1} A & I
\end{array}\right],
$$

从而

$$
\sigma(H)=\sigma\left(H_{0}\right), \quad \sigma_{p}(H)=\sigma_{p}\left(H_{0}\right), \quad \sigma_{r}(H)=\sigma_{r}\left(H_{0}\right), \quad \sigma_{c}(H)=\sigma_{c}\left(H_{0}\right),
$$

其中

$$
H_{0}=\left[\begin{array}{cc}
0 & B \\
C+B^{-1} A^{2} & 0
\end{array}\right]
$$

注意到

$$
B\left(C+B^{-1} A^{2}\right)=A^{2}+B C, \quad\left(C+B^{-1} A^{2}\right) B=A^{* 2}+C B,
$$

根据文献 [19] 中的结论便证得 (2)-(5).

注 2.2 当 $A$ 为有界算子时, 引理 2.2 的结论 (1) 成立, 不需要 $B^{-1} A$ 为自伴算子的条件. 如果 $A$ 有界且 $B^{-1} A$ 为自伴算子, 则 $\sigma(H), \rho(H)$ 均关于虚轴及原点对称, 从而关于实轴对称. 另外, 在引 理 2.2 的条件下, $\sigma_{p}(H)$ 关于原点对称, 它关于虚轴对称的充要条件为 $\sigma_{r}(H)=\emptyset$, 此时也关于实轴对 称.

引理 2.3 在假设 (i)-(ii) 下, 下述结论成立:

若

$$
\left[\begin{array}{c}
p \\
\lambda B^{-1} p-B^{-1} A p
\end{array}\right] \text { 和 }\left[\begin{array}{c}
u \\
\left(\lambda B^{-1}-B^{-1} A\right) u+B^{-1} p
\end{array}\right]
$$

分别为 $H$ 相应于本征值 $\lambda$ 的基本本征函数和 Jordan 型本征函数, 则

$$
\left[\begin{array}{c}
-p \\
\lambda B^{-1} p+B^{-1} A p
\end{array}\right] \text { 和 }\left[\begin{array}{c}
u \\
-\left(\lambda B^{-1}+B^{-1} A\right) u-B^{-1} p
\end{array}\right]
$$

分别为 $H$ 相应于 $-\lambda$ 的基本本征函数和 Jordan 型本征函数. 这里 $p$ 和 $u$ 为下列方程的非零解

$$
\begin{aligned}
& C p=\lambda^{2} B^{-1} p-B^{-1} A^{2} p, \\
& C u=\left(\lambda^{2} B^{-1}-B^{-1} A^{2}\right) u+2 \lambda B^{-1} p .
\end{aligned}
$$

证明 设 $[p, q]^{T}$ 与 $[u, v]^{T}$ 分别为 $H$ 相应于 $\lambda$ 的基本本征函数和 Jordan 型本征函数, 则

$$
\left[\begin{array}{cc}
A & B \\
C & -A^{*}
\end{array}\right]\left[\begin{array}{l}
p \\
q
\end{array}\right]=\lambda\left[\begin{array}{l}
p \\
q
\end{array}\right]
$$

且

$$
\left[\begin{array}{cc}
A & B \\
C & -A^{*}
\end{array}\right]\left[\begin{array}{l}
u \\
v
\end{array}\right]=\lambda\left[\begin{array}{l}
u \\
v
\end{array}\right]+\left[\begin{array}{l}
p \\
q
\end{array}\right] .
$$

求解方程 (2.6) 并结合假设 (i)-(ii), 我们有

$$
q=\lambda B^{-1} p-B^{-1} A p
$$

60 
且

$$
C p=\lambda^{2} B^{-1} p+\lambda\left(A^{*} B^{-1}-B^{-1} A\right) p-A^{*} B^{-1} A p=\lambda^{2} B^{-1} p-B^{-1} A^{2} p .
$$

将方程 (2.8) 代入 (2.7) 中, 经求解得到

$$
v=\left(\lambda B^{-1}-B^{-1} A\right) u+B^{-1} p
$$

及

$$
\begin{aligned}
C u & =\left(\lambda^{2} B^{-1}-A^{*} B^{-1} A\right) u+\left(A^{*} B^{-1}-B^{-1} A\right)(\lambda u+p)+2 \lambda B^{-1} p \\
& =\left(\lambda^{2} B^{-1}-B^{-1} A^{2}\right) u+2 \lambda B^{-1} p .
\end{aligned}
$$

结合前面的公式, 方程 (2.8) 与定义 2.3 , 易知 $p$ 和 $u$ 都是非零的.

另外, 经计算

$$
\begin{aligned}
{\left[\begin{array}{cc}
A & B \\
C & -A^{*}
\end{array}\right]\left[\begin{array}{c}
-p \\
\lambda B^{-1} p+B^{-1} A p
\end{array}\right] } & =\left[\begin{array}{c}
\lambda p \\
-C p-B^{-1} A^{2} p-\lambda B^{-1} A p
\end{array}\right] \\
& =\left[\begin{array}{c}
\lambda p \\
-\lambda^{2} B^{-1} p-\lambda B^{-1} A p
\end{array}\right]=-\lambda\left[\begin{array}{c}
-p \\
\lambda B^{-1} p+B^{-1} A p
\end{array}\right]
\end{aligned}
$$

且

$$
\begin{aligned}
& {\left[\begin{array}{cc}
A & B \\
C & -A^{*}
\end{array}\right]\left[\begin{array}{c}
u \\
-\left(\lambda B^{-1}+B^{-1} A\right) u-B^{-1} p
\end{array}\right]} \\
& \quad=\left[\begin{array}{c}
-\lambda u-p \\
\left(C+\lambda B^{-1} A+B^{-1} A^{2}\right) u+B^{-1} A p
\end{array}\right]=\left[\begin{array}{c}
u \\
\left(\lambda^{2} B^{-1}+\lambda B^{-1} A\right) u+2 \lambda B^{-1} p+B^{-1} A p
\end{array}\right] \\
& \quad=-\lambda\left[\begin{array}{c}
-\lambda u-p \\
-p \\
-\left(\lambda B^{-1}+B^{-1} A\right) u-B^{-1} p
\end{array}\right]+\left[\begin{array}{c}
-p \\
\lambda B^{-1} p+B^{-1} A p
\end{array}\right] .
\end{aligned}
$$

引理 2.3 证毕.

由引理 2.2 和引理 2.3 知 $H$ 的本征值仅正负成对出现, 记为 $\left\{\lambda_{ \pm n}\right\}_{n=1}^{+\infty}\left(\lambda_{-n}=-\lambda_{n}\right)$. 于是 $H$ 相 应于本征值 $\lambda_{n}$ 的基本本征函数 $Q_{n}^{0}$ 和 Jordan 型本征函数 $Q_{n}^{1}$ 具有如下形式

$$
\begin{aligned}
& Q_{n}^{0}=\left[\begin{array}{l}
p_{n} \\
q_{n}
\end{array}\right]=\left[\begin{array}{c}
p_{n} \\
\lambda_{n} B^{-1} p_{n}-B^{-1} A p_{n}
\end{array}\right], \\
& Q_{n}^{1}=\left[\begin{array}{c}
u_{n} \\
v_{n}
\end{array}\right]=\left[\begin{array}{c}
u_{n} \\
\left(\lambda_{n} B^{-1}-B^{-1} A\right) u_{n}+B^{-1} p_{n}
\end{array}\right] .
\end{aligned}
$$

符号 $Q_{-n}^{0}$ 和 $Q_{-n}^{1}$ 表示 $H$ 相应于本征值 $\lambda_{-n}$ 的基本本征函数和 Jordan 型本征函数, 它们具有如下 形式:

$$
Q_{-n}^{0}=\left[\begin{array}{c}
p_{-n} \\
q_{-n}
\end{array}\right]=\left[\begin{array}{c}
-p_{n} \\
\lambda_{n} B^{-1} p_{n}+B^{-1} A p_{n}
\end{array}\right]
$$




$$
Q_{-n}^{1}=\left[\begin{array}{c}
u_{-n} \\
v_{-n}
\end{array}\right]=\left[\begin{array}{c}
u_{n} \\
-\left(\lambda_{n} B^{-1}+B^{-1} A\right) u_{n}-B^{-1} p_{n}
\end{array}\right]
$$

容易看出

$$
p_{-n}=-p_{n}, \quad u_{-n}=u_{n}(n=1,2, \ldots) .
$$

引理 2.4 在假设 (i)-(ii) 下, 若 $\lambda_{n}^{2}-\bar{\lambda}_{m}^{2} \neq 0$, 则 $\left\{p_{n}\right\}_{n=1}^{+\infty}$ 和 $\left\{u_{n}\right\}_{n=1}^{+\infty}$ 之间满足下列正交关系:

$$
\left\langle B^{-1} p_{n}, p_{m}\right\rangle=\left\langle B^{-1} u_{n}, p_{m}\right\rangle=0 .
$$

证明 根据引理 2.1 , 当 $\lambda_{n}+\bar{\lambda}_{m} \neq 0$ 时, 我们有

$$
\left\langle Q_{n}^{0}, J Q_{m}^{0}\right\rangle=\left\langle Q_{n}^{0}, J Q_{m}^{1}\right\rangle=\left\langle Q_{n}^{1}, J Q_{m}^{1}\right\rangle=0
$$

通过直接计算, 得到

$$
\begin{aligned}
& \left\langle Q_{n}^{0}, J Q_{m}^{0}\right\rangle=\left(\bar{\lambda}_{m}-\lambda_{n}\right)\left\langle B^{-1} p_{n}, p_{m}\right\rangle \\
& \left\langle Q_{n}^{0}, J Q_{m}^{1}\right\rangle=\left(\bar{\lambda}_{m}-\lambda_{n}\right)\left\langle B^{-1} p_{n}, u_{m}\right\rangle+\left\langle B^{-1} p_{n}, p_{m}\right\rangle, \\
& \left\langle Q_{n}^{1}, J Q_{m}^{1}\right\rangle=\left(\bar{\lambda}_{m}-\lambda_{n}\right)\left\langle B^{-1} u_{n}, u_{m}\right\rangle+\left\langle B^{-1} u_{n}, p_{m}\right\rangle-\left\langle B^{-1} p_{n}, u_{m}\right\rangle .
\end{aligned}
$$

故当 $\lambda_{n}^{2}-\bar{\lambda}_{m}^{2} \neq 0$ 时, 公式 (2.11) 成立. 引理 2.4 证毕.

引理 2.5 ${ }^{[20]}$ Hilbert 空间 $L^{2}(0, l)$ 中的正交函数系 $\left\{\sin \frac{n \pi y}{l}\right\}_{n=1}^{+\infty}$ 在 $L^{2}(0, l)$ 空间中是完备的, 即对于任意的 $\phi \in L^{2}(0, l)$, 相应的 Fourier 级数

$$
\frac{2}{l} \sum_{n=1}^{+\infty}\left(\int_{0}^{l} \phi(\xi) \sin \frac{n \pi \xi}{l} d \xi\right) \sin \frac{n \pi y}{l}
$$

按 $L^{2}(0, l)$ 的范数收玫于 $\phi$.

\section{3 主要结果}

下面的定理是本文的主要结果, 它描述了 Hamilton 算子 $H$ 广义本征函数系的完备性.

定理 3.1 在假设 (i)-(ii) 条件下, 若 $H$ 还满足

(iii) $\left\langle B^{-1} p_{n}, u_{n}\right\rangle=-\lambda_{n}\left\langle B^{-1} u_{n}, u_{n}\right\rangle, n=1,2, \ldots$;

(iv) $\left\langle B^{-1} p_{n}, p_{n}\right\rangle=0, n=1,2, \ldots$;

(v) $\left\langle B^{-1} u_{n}, u_{n}\right\rangle \neq 0, n=1,2, \ldots$,

则 $H$ 的由 $\left\{Q_{n}^{0}\right\}_{n= \pm 1}^{ \pm \infty}$ 和 $\left\{Q_{n}^{1}\right\}_{n= \pm 1}^{ \pm \infty}$ 构成的广义本征函数系在 $X \times X$ 中 Cauchy 主值意义下完备 当且仅当函数系 $\left\{p_{n}\right\}_{n=1}^{+\infty} \cup\left\{u_{n}\right\}_{n=1}^{+\infty}$ 在 $X$ 中完备.

证明 根据 $(2.5 \mathrm{~b})$ 式, $p_{n}$ 和 $u_{n}$ 满足下述方程

$$
C u_{n}+A^{*} B^{-1} A u_{n}=\lambda_{n}^{2} B^{-1} u_{n}+2 \lambda_{n} B^{-1} p_{n} .
$$

用 $u_{n}$ 在上式两端作内积, 得到

$$
\left\langle C u_{n}, u_{n}\right\rangle+\left\langle B^{-1} A u_{n}, A u_{n}\right\rangle=\lambda_{n}^{2}\left\langle B^{-1} u_{n}, u_{n}\right\rangle+2 \lambda_{n}\left\langle B^{-1} p_{n}, u_{n}\right\rangle .
$$

62 
由 (iii) 和 (v), 我们有

$$
\lambda_{n}^{2}=-\frac{\left\langle C u_{n}, u_{n}\right\rangle+\left\langle B^{-1} A u_{n}, A u_{n}\right\rangle}{\left\langle B^{-1} u_{n}, u_{n}\right\rangle} \in \mathbb{R}
$$

于是 $\lambda_{n} \in i \mathbb{R} \cup \mathbb{R}$.

因为 $\sigma_{p}(H) \subset i \mathbb{R} \cup \mathbb{R}$, 故 $\lambda_{n}+\bar{\lambda}_{m}=0$ 仅包含两种情形: $\lambda_{m}=-\lambda_{n} \in \mathbb{R}$ 及 $\lambda_{m}=\lambda_{n} \in i \mathbb{R}$, 设这两 种情形分别对应于 $m=-n$ 及 $m=n$. 利用引理 2.1、引理 2.4 和公式 (2.9)-(2.12) 并结合 (iv), 可得

$$
\begin{aligned}
& \left\langle Q_{n}^{0}, J Q_{m}^{0}\right\rangle=\left\langle Q_{n}^{1}, J Q_{m}^{1}\right\rangle=0, \quad n, m= \pm 1, \pm 2, \ldots, \\
& \left\langle Q_{n}^{0}, J Q_{m}^{1}\right\rangle=\left\{\begin{array}{cl}
0, & n \neq-m, \\
2 \lambda_{n}^{2}\left\langle B^{-1} u_{n}, u_{n}\right\rangle, & n=-m,
\end{array}\right.
\end{aligned}
$$

且

$$
\begin{aligned}
& \left\langle Q_{n}^{0}, J Q_{m}^{0}\right\rangle=\left\langle Q_{n}^{1}, J Q_{m}^{1}\right\rangle=0, \quad n, m= \pm 1, \pm 2, \ldots, \\
& \left\langle Q_{n}^{0}, J Q_{m}^{1}\right\rangle=\left\{\begin{array}{cl}
0, & n \neq m, \\
2 \lambda_{n}^{2}\left\langle B^{-1} u_{n}, u_{n}\right\rangle, & n=m .
\end{array}\right.
\end{aligned}
$$

由于 $p_{n}$ 和 $u_{n}$ 分别为 $Q_{n}^{0}$ 和 $Q_{n}^{1}$ 的第一分量, 结合方程 (2.10) 知必要性显然. 对于充分性, 只需 要证明对于任意的

$$
f(x)=\left(\begin{array}{l}
f_{1} \\
f_{2}
\end{array}\right) \in X \times X,
$$

都存在常数序列 $\left\{f_{ \pm n}^{0}\right\}_{n=1}^{+\infty},\left\{f_{ \pm n}^{1}\right\}_{n=1}^{+\infty}$ 满足

$$
\begin{aligned}
f(x) & =\lim _{N \rightarrow+\infty} \sum_{n=1}^{N}\left(f_{n}^{0} Q_{n}^{0}+f_{n}^{1} Q_{n}^{1}+f_{-n}^{0} Q_{-n}^{0}+f_{-n}^{1} Q_{-n}^{1}\right) \\
& =\sum_{n=1}^{+\infty}\left(f_{n}^{0} Q_{n}^{0}+f_{n}^{1} Q_{n}^{1}+f_{-n}^{0} Q_{-n}^{0}+f_{-n}^{1} Q_{-n}^{1}\right) .
\end{aligned}
$$

事实上, 若 $\lambda_{n} \in \mathbb{R}$, 根据引理 2.1 及 (3.1) 式, 取

$$
f_{ \pm n}^{1}=-\frac{\left\langle f(x), J Q_{\mp n}^{0}\right\rangle}{2 \lambda_{n}^{2}\left\langle B^{-1} u_{n}, u_{n}\right\rangle}, \quad f_{ \pm n}^{0}=\frac{\left\langle f(x), J Q_{\mp n}^{1}\right\rangle}{2 \lambda_{n}^{2}\left\langle B^{-1} u_{n}, u_{n}\right\rangle} \quad n=1,2, \ldots
$$

若 $\lambda_{n} \in i \mathbb{R}$, 根据引理 2.1 及 $(3.2)$ 式, 取

$$
f_{ \pm n}^{1}=-\frac{\left\langle f(x), J Q_{ \pm n}^{0}\right\rangle}{2 \lambda_{n}^{2}\left\langle B^{-1} u_{n}, u_{n}\right\rangle}, \quad f_{ \pm n}^{0}=\frac{\left\langle f(x), J Q_{ \pm n}^{1}\right\rangle}{2 \lambda_{n}^{2}\left\langle B^{-1} u_{n}, u_{n}\right\rangle} \quad n=1,2, \ldots
$$

利用 (3.4) 和 (3.5), 不论 $\lambda_{n} \in \mathbb{R}$ 还是 $\lambda_{n} \in i \mathbb{R}$, 我们总有

$$
\begin{aligned}
\sum_{n=1}^{N}\left(f_{n}^{0} Q_{n}^{0}\right. & \left.+f_{n}^{1} Q_{n}^{1}+f_{-n}^{0} Q_{-n}^{0}+f_{-n}^{1} Q_{-n}^{1}\right) \\
& =\sum_{n=1}^{N} \frac{1}{\alpha_{n}}\left[\begin{array}{c}
\left(\left\langle f_{1}, B^{-1} p_{n}\right\rangle+\bar{\lambda}_{n}\left\langle f_{1}, B^{-1} u_{n}\right\rangle\right) p_{n}+\lambda_{n}\left\langle f_{1}, B^{-1} p_{n}\right\rangle u_{n} \\
\left(\left\langle f_{2}, p_{n}\right\rangle+\bar{\lambda}_{n}\left\langle f_{2}, u_{n}\right\rangle\right) B^{-1} p_{n}+\lambda_{n}\left\langle f_{2}, p_{n}\right\rangle B^{-1} u_{n}+\eta_{n}
\end{array}\right],
\end{aligned}
$$


其中 $\alpha_{n}=-\left|\lambda_{n}\right|^{2}\left\langle B^{-1} u_{n}, u_{n}\right\rangle$ 且

$$
\begin{aligned}
\eta_{n}= & \left(\left\langle f_{1}, B^{-1} A p_{n}\right\rangle+\bar{\lambda}_{n}\left\langle f_{1}, B^{-1} A u_{n}\right\rangle\right) B^{-1} p_{n}+\lambda_{n}\left\langle f_{1}, B^{-1} A p_{n}\right\rangle B^{-1} u_{n} \\
& -\lambda_{n}\left\langle f_{1}, B^{-1} p_{n}\right\rangle B^{-1} A u_{n}-\left(\left\langle f_{1}, B^{-1} p_{n}\right\rangle+\bar{\lambda}_{n}\left\langle f_{1}, B^{-1} u_{n}\right\rangle\right) B^{-1} A p_{n} .
\end{aligned}
$$

因为函数系 $\left\{p_{n}\right\}_{n=1}^{+\infty} \cup\left\{u_{n}\right\}_{n=1}^{+\infty}$ 在 $X$ 中完备且 $B^{-1}$ 有界, 故 $\left\{B^{-1} p_{n}\right\}_{n=1}^{+\infty} \cup\left\{B^{-1} u_{n}\right\}_{n=1}^{+\infty}$ 在 $X$ 中 完备. 于是存在常数序列 $\left\{C_{ \pm n}^{1}\right\}_{n=1}^{+\infty}$ 和 $\left\{C_{ \pm n}^{2}\right\}_{n=1}^{+\infty}$ 满足

$$
\begin{aligned}
& f_{1}=\lim _{N \rightarrow+\infty} \sum_{n=1}^{N}\left(C_{n}^{1} p_{n}+C_{-n}^{1} u_{n}\right)=\sum_{n=1}^{+\infty}\left(C_{n}^{1} p_{n}+C_{-n}^{1} u_{n}\right), \\
& f_{2}=\lim _{N \rightarrow+\infty} \sum_{n=1}^{N}\left(C_{n}^{2} B^{-1} p_{n}+C_{-n}^{2} B^{-1} u_{n}\right)=\sum_{n=1}^{+\infty}\left(C_{n}^{2} B^{-1} p_{n}+C_{-n}^{2} B^{-1} u_{n}\right) .
\end{aligned}
$$

分别用 $B^{-1} p_{n}$ 和 $B^{-1} u_{n}$ 与式 (3.8) 的第一式两端作内积, 结合引理 2.4 , 我们有

$$
\left\langle f_{1}, B^{-1} p_{n}\right\rangle=\left\langle\sum_{n=1}^{+\infty} C_{n}^{1} p_{n}+C_{-n}^{1} u_{n}, B^{-1} p_{n}\right\rangle=C_{-n}^{1}\left\langle B^{-1} u_{n}, p_{n}\right\rangle=-\bar{\lambda}_{n}\left\langle B^{-1} u_{n}, u_{n}\right\rangle C_{-n}^{1}
$$

且

$$
\left\langle f_{1}, B^{-1} u_{n}\right\rangle=\left\langle\sum_{n=1}^{+\infty} C_{n}^{1} p_{n}+C_{-n}^{1} u_{n}, B^{-1} u_{n}\right\rangle=\left(C_{-n}^{1}-\lambda_{n} C_{n}^{1}\right)\left\langle B^{-1} u_{n}, u_{n}\right\rangle
$$

于是

$$
C_{-n}^{1}=-\frac{\left\langle f_{1}, B^{-1} p_{n}\right\rangle}{\bar{\lambda}_{n}\left\langle B^{-1} u_{n}, u_{n}\right\rangle}, \quad C_{n}^{1}=\frac{-1}{\lambda_{n}\left\langle B^{-1} u_{n}, u_{n}\right\rangle}\left(\left\langle f_{1}, B^{-1} u_{n}\right\rangle+\frac{1}{\bar{\lambda}_{n}}\left\langle f_{1}, B^{-1} p_{n}\right\rangle\right) .
$$

类似地, 分别用 $p_{n}$ 和 $u_{n}$ 在式 (3.8) 的第二式两端作内积, 结合引理 2.4, 得

$$
\left\langle f_{2}, p_{n}\right\rangle=\left\langle\sum_{n=1}^{+\infty}\left(C_{n}^{2} B^{-1} p_{n}+C_{-n}^{2} B^{-1} u_{n}\right), p_{n}\right\rangle=C_{-n}^{2}\left\langle B^{-1} u_{n}, p_{n}\right\rangle=-\bar{\lambda}_{n}\left\langle B^{-1} u_{n}, u_{n}\right\rangle C_{-n}^{2}
$$

且

$$
\left\langle f_{2}, u_{n}\right\rangle=\left\langle\sum_{n=1}^{+\infty}\left(C_{n}^{2} B^{-1} p_{n}+C_{-n}^{2} B^{-1} u_{n}\right), u_{n}\right\rangle=\left(C_{-n}^{2}-\lambda_{n} C_{n}^{2}\right)\left\langle B^{-1} u_{n}, u_{n}\right\rangle .
$$

进而

$$
C_{-n}^{2}=-\frac{\left\langle f_{2}, p_{n}\right\rangle}{\bar{\lambda}_{n}\left\langle B^{-1} u_{n}, u_{n}\right\rangle}, \quad C_{n}^{2}=\frac{-1}{\lambda_{n}\left\langle B^{-1} u_{n}, u_{n}\right\rangle}\left(\left\langle f_{2}, u_{n}\right\rangle+\frac{1}{\bar{\lambda}_{n}}\left\langle f_{2}, p_{n}\right\rangle\right) .
$$

根据 (3.9)-(3.10), 式 (3.8) 可改写为

$$
\begin{aligned}
& f_{1}=\lim _{N \rightarrow+\infty} \sum_{n=1}^{N} \frac{1}{\alpha_{n}}\left\{\left(\left\langle f_{1}, B^{-1} p_{n}\right\rangle+\bar{\lambda}_{n}\left\langle f_{1}, B^{-1} u_{n}\right\rangle\right) p_{n}+\lambda_{n}\left\langle f_{1}, B^{-1} p_{n}\right\rangle u_{n}\right\}, \\
& f_{2}=\lim _{N \rightarrow+\infty} \sum_{n=1}^{N} \frac{1}{\alpha_{n}}\left\{\left(\left\langle f_{2}, p_{n}\right\rangle+\bar{\lambda}_{n}\left\langle f_{2}, u_{n}\right\rangle\right) B^{-1} p_{n}+\lambda_{n}\left\langle f_{2}, p_{n}\right\rangle B^{-1} u_{n}\right\} .
\end{aligned}
$$

用同样的方法, 我们可导出 $B^{-1} A f_{1}$ 有下列表示形式

$$
B^{-1} A f_{1}=\lim _{N \rightarrow+\infty} \sum_{n=1}^{N} \frac{1}{\alpha_{n}}\left\{\left(\left\langle B^{-1} A f_{1}, p_{n}\right\rangle+\bar{\lambda}_{n}\left\langle B^{-1} A f_{1}, u_{n}\right\rangle\right) B^{-1} p_{n}+\lambda_{n}\left\langle B^{-1} A f_{1}, p_{n}\right\rangle B^{-1} u_{n}\right\} .
$$


注意到 $B^{-1} A$ 有界, 利用方程 (3.11a) 有

$$
B^{-1} A f_{1}=\lim _{N \rightarrow+\infty} \sum_{n=1}^{N} \frac{1}{\alpha_{n}}\left\{\left(\left\langle f_{1}, B^{-1} p_{n}\right\rangle+\bar{\lambda}_{n}\left\langle f_{1}, B^{-1} u_{n}\right\rangle\right) B^{-1} A p_{n}+\lambda_{n}\left\langle f_{1}, B^{-1} p_{n}\right\rangle B^{-1} A u_{n}\right\} .
$$

利用上述两式并结合方程 (3.7), 可得

$$
\lim _{N \rightarrow+\infty} \sum_{n=1}^{N} \frac{\eta_{n}}{\alpha_{n}}=0 .
$$

根据 (3.6), (3.11) 及 $(3.12)$ 式, 我们有

$$
\begin{aligned}
\lim _{N \rightarrow+\infty} & \sum_{n=1}^{N}\left(f_{n}^{0} Q_{n}^{0}+f_{n}^{1} Q_{n}^{1}+f_{-n}^{0} Q_{-n}^{0}+f_{-n}^{1} Q_{-n}^{1}\right) \\
\quad & =\sum_{n=1}^{\infty} \frac{1}{\alpha_{n}}\left(\begin{array}{c}
\left(\left\langle f_{1}, B^{-1} p_{n}\right\rangle+\bar{\lambda}_{n}\left\langle f_{1}, B^{-1} u_{n}\right\rangle\right) p_{n}+\lambda_{n}\left\langle f_{1}, B^{-1} p_{n}\right\rangle u_{n} \\
\left(\left\langle f_{2}, p_{n}\right\rangle+\bar{\lambda}_{n}\left\langle f_{2}, u_{n}\right\rangle\right) B^{-1} p_{n}+\lambda_{n}\left\langle f_{2}, p_{n}\right\rangle B^{-1} u_{n}+\eta_{n}
\end{array}\right)=\left(\begin{array}{c}
f_{1} \\
f_{2}
\end{array}\right)=f(x) .
\end{aligned}
$$

于是 (3.3) 式成立.

定理 3.1 证毕.

下面给出 Hamilton 系统 (2.1) 的一般解. 根据定理 3.1 和解的叠加原理, 方程 (2.1) 的解具有如下 形式:

$$
U(t, y)=\sum_{n=1}^{\infty}\left\{T_{n}^{0}(t) Q_{n}^{0}(y)+T_{-n}^{0}(t) Q_{-n}^{0}(y)+T_{n}^{1}(t) Q_{n}^{1}(y)+T_{-n}^{1}(t) Q_{-n}^{1}(y)\right\} .
$$

将 (3.13) 式代入 (2.1), 可得

$$
T_{n}^{1}(t)=c_{n}^{1} e^{\lambda_{n} t}, \quad T_{n}^{0}(t)=\left(c_{n}^{0}+t c_{n}^{1}\right) e^{\lambda_{n} t}(n= \pm 1, \pm 2, \cdots),
$$

其中 $c_{n}^{0}, c_{n}^{1}$ 为任意常数, 它们由侧边 $t$ 的边界条件确定.

将 (3.14) 式代入 (3.13) 式中, 得 Hamilton 系统 (2.1) 的一般解为:

$$
\begin{aligned}
U(t, y)= & \sum_{n=1}^{\infty}\left\{c_{n}^{0} e^{\lambda_{n} t} Q_{n}^{0}(y)+c_{n}^{1} e^{\lambda_{n} t}\left(Q_{n}^{1}(y)+t Q_{n}^{0}(y)\right)\right. \\
& \left.+c_{-n}^{0} e^{-\lambda_{n} t} Q_{-n}^{0}(y)+c_{-n}^{1} e^{-\lambda_{n} t}\left(Q_{-n}^{1}(y)+t Q_{-n}^{0}(y)\right)\right\} .
\end{aligned}
$$

\section{4 在弹性力学板弯曲方程中的应用}

本节利用定理 3.1 证明对边简支的矩形薄板问题导出的无穷维 Hamilton 算子 $H$ 广义本征函数 系的完备性.

不失一般性, 在区域 $\{(x, y) \mid 0 \leqslant x \leqslant h, 0 \leqslant y \leqslant 1)\}$ 内考虑如下齐次方程

$$
D\left(\frac{\partial^{2}}{\partial x^{2}}+\frac{\partial^{2}}{\partial y^{2}}\right)^{2} w=0
$$

及对边简支边界条件

$$
w=0, \quad \frac{\partial^{2} w}{\partial y^{2}}=0(y=0 \text { 及 } y=1) .
$$


引入板的弯矩函数

$$
m=-D\left(\frac{\partial^{2} w}{\partial x^{2}}+\frac{\partial^{2} w}{\partial y^{2}}\right)
$$

则方程 (4.1) 可改写为

$$
\left(\frac{\partial^{2}}{\partial x^{2}}+\frac{\partial^{2}}{\partial y^{2}}\right) m=0
$$

为得到方程 (4.1) 对应的 Hamilton 系统, 令

$$
p=-\frac{\partial m}{\partial x}-w, \quad q=\frac{\partial w}{\partial x},
$$

结合 (4.3)-(4.5) 式, 我们有

$$
\left\{\begin{array}{l}
\frac{\partial w}{\partial x}=q \\
\frac{\partial m}{\partial x}=-w-p \\
\frac{\partial p}{\partial x}=-\frac{\partial^{2} m}{\partial x^{2}}-\frac{\partial w}{\partial x}=-\frac{\partial^{2}(-m)}{\partial y^{2}}-q \\
\frac{\partial q}{\partial x}=\frac{\partial^{2} w}{\partial x^{2}}=-\frac{\partial^{2} w}{\partial y^{2}}-\frac{m}{D}
\end{array}\right.
$$

上述方程组可写成如下无穷维 Hamilton 系统:

$$
\frac{\partial U}{\partial x}=\left[\begin{array}{cccc}
0 & 0 & 0 & 1 \\
1 & 0 & 1 & 0 \\
0 & -\frac{\partial^{2}}{\partial y^{2}} & 0 & -1 \\
-\frac{\partial^{2}}{\partial y^{2}} & \frac{1}{D} & 0 & 0
\end{array}\right] U=H U
$$

其中 $U=[w,-m, p, q]^{T}$ 为全状态变量. 这里用变量 $x$ 模拟 Hamilton 系统中的时间变量.

记

$$
A=\left[\begin{array}{ll}
0 & 0 \\
1 & 0
\end{array}\right], \quad B=\left[\begin{array}{ll}
0 & 1 \\
1 & 0
\end{array}\right], \quad C=\left[\begin{array}{cc}
0 & -\frac{d^{2}}{d y^{2}} \\
-\frac{d^{2}}{d y^{2}} & \frac{1}{D}
\end{array}\right],
$$

结合边界条件 (4.2), 可得如下无穷维 Hamilton 算子:

$$
\begin{aligned}
& H=\left[\begin{array}{cc}
A & B \\
C & -A^{*}
\end{array}\right]: \mathcal{D}(H) \subset X \times X \rightarrow X \times X, \\
& \mathcal{D}(H)=\left\{\begin{array}{l|l}
U(y) \in X & \left.\begin{array}{l}
w(0)=w(1)=0, m(0)=m(1)=0, w^{\prime}, \\
m^{\prime} \text { 绝对连续, } w^{\prime}, w^{\prime \prime}, m^{\prime}, m^{\prime \prime} \in L^{2}(0,1)
\end{array}\right\},
\end{array},\right.
\end{aligned}
$$

其中 $X=L^{2}(0,1) \times L^{2}(0,1)$.

直接计算可得算子 $(4.7)$ 的本征值为 $\lambda_{n}=n \pi(n= \pm 1, \pm 2, \ldots)$, 相应于 $\lambda_{n}$ 的基本本征函数和 Jordan 型本征函数为:

$$
\begin{aligned}
& Q_{n}^{0}=\left[\frac{1}{\lambda_{n}} \sin \lambda_{n} y, 0,-\frac{1}{\lambda_{n}} \sin \lambda_{n} y, \sin \lambda_{n} y\right]^{T}, \\
& Q_{n}^{1}=\left[-\frac{1}{2 \lambda_{n}^{2}} \sin \lambda_{n} y, D \sin \lambda_{n} y,\left(\lambda_{n} D+\frac{1}{2 \lambda_{n}^{2}}\right) \sin \lambda_{n} y,\left(\frac{1}{2 \lambda_{n}}-1\right) \sin \lambda_{n} y\right]^{T},
\end{aligned}
$$

66 
其中 $n= \pm 1, \pm 2, \ldots$,

$$
p_{n}=\left[\frac{1}{\lambda_{n}} \sin \lambda_{n} y, 0\right]^{T}, \quad u_{n}=\left[-\frac{1}{2 \lambda_{n}^{2}} \sin \lambda_{n} y, D \sin \lambda_{n} y\right]^{T} .
$$

不难验证算子 (4.7) 满足假设 (i)-(ii) 且

$$
\begin{aligned}
& \left\langle B^{-1} p_{n}, u_{n}\right\rangle=\frac{D}{\lambda_{n}} \int_{0}^{1} \sin ^{2} \lambda_{n} y d y=\frac{D}{2 \lambda_{n}}, \\
& \left\langle B^{-1} u_{n}, u_{n}\right\rangle=-\frac{D}{\lambda_{n}^{2}} \int_{0}^{1} \sin ^{2} \lambda_{n} y d y=-\frac{D}{2 \lambda_{n}^{2}} \neq 0, \\
& \left\langle B^{-1} p_{n}, p_{n}\right\rangle=0 .
\end{aligned}
$$

因而也满足定理 3.1 中的假设 (iii)-(v). 根据定理 3.1, 要说明算子 (4.7) 的广义本征函数系是完 备的, 只需验证 $\left\{p_{n}\right\}_{n=1}^{+\infty} \cup\left\{u_{n}\right\}_{n=1}^{+\infty}$ 在 Hilbert 空间 $X$ 中完备. 为此, 只需证明对于任意的 $g(x)=$ $\left[\begin{array}{l}g_{1}(x) \\ g_{2}(x)\end{array}\right] \in X$, 存在常数序列 $\left\{c_{n}^{p}\right\}_{n=1}^{+\infty},\left\{c_{n}^{u}\right\}_{n=1}^{+\infty}$ 满足

$$
g(x)=\sum_{n=1}^{+\infty}\left(c_{n}^{p} p_{n}+c_{n}^{u} u_{n}\right)
$$

事实上, 据引理 2.4 , 取

$$
\begin{aligned}
& c_{n}^{u}=-\frac{\left\langle g(x), B^{-1} p_{n}\right\rangle}{\lambda_{n}\left\langle B^{-1} u_{n}, u_{n}\right\rangle}=\frac{2 \lambda_{n}}{D} \int_{0}^{1} g_{2}(\xi) \sin \lambda_{n} \xi d \xi, \\
& c_{n}^{p}=\frac{c_{n}^{u}}{\lambda_{n}}-\frac{\left\langle g(x), B^{-1} u_{n}\right\rangle}{\lambda_{n}\left\langle B^{-1} u_{n}, u_{n}\right\rangle}=2 \lambda_{n} \int_{0}^{1} g_{1}(\xi) \sin \lambda_{n} \xi d \xi+\frac{2}{\lambda_{n} D} \int_{0}^{1} g_{2}(\xi) \sin \lambda_{n} \xi d \xi,
\end{aligned}
$$

代入 (4.8) 式的右端, 可得

$$
\sum_{n=1}^{+\infty}\left(c_{n}^{p} p_{n}+c_{n}^{u} u_{n}\right)=\left[\begin{array}{c}
2 \sum_{n=1}^{+\infty}\left(\int_{0}^{b} g_{1}(\xi) \sin \lambda_{n} \xi d \xi\right) \sin \lambda_{n} y \\
2 \sum_{n=1}^{+\infty}\left(\int_{0}^{b} g_{2}(\xi) \sin \lambda_{n} \xi d \xi\right) \sin \lambda_{n} y
\end{array}\right] .
$$

显然上述向量的第一分量和第二分量分别为 $g_{1}(x)$ 和 $g_{2}(x)$ 按 $L^{2}(0,1)$ 中的正交函数系 $\left\{\sin \lambda_{n} y\right\}_{n=1}^{+\infty}$ 展开所得的 Fourier 级数, 由引理 2.5 知 (4.9) 收玫于 $g(x)$. 根据定理 3.1 知, 算子 (4.7) 的广义本征函数系在 $X \times X$ 中 Cauchy 主值意义下完备.

\section{5 结论}

力学中的许多问题是非自伴的, 非自伴问题较难解决. 为克服自伴性的限制, 推广 Hilbert-Schmidt 展开定理, 诸多学者运用 Hamilton 体系下的分离变量法求解了许多应用力学问题, 这必然导向无穷维 Hamilton 算子的本征值问题, 但相应的数学理论还没有系统建立. 本文得到了一类无穷维 Hamilton 算子的广义本征函数系在 Cauchy 主值意义下的完备性判别准则, 并应用于板弯曲方程. 相应结果约 化了问题的维数, 为应用辛体系方法求解某些力学问题提供了理论保障.

致谢引理 2.2 根据审稿人的评阅意见完成, 作者对审稿人的细致评阅和有益建议表示衰心感谢. 


\section{参考文献}

1 Chen Y, Zheng Y, Zhang H Q. The Hamiltonian equations in some mathematics and physics problems. Appl Math Mech (English Ed), 2003, 24: 22-27

2 Ren W X and Alatancang. An algorithm and its application for obtaining some kind of innite-dimensional Hamiltonian canonical formulation. Commun Theor Phys (Beijing), 2007, 16: 3154-3160

3 阿拉坦仓, 张鸿庆, 钟万氻思. 一类偏微分方程的无穷维 Hamilton 正则表示. 力学学报, 2000, 31: 347-357

4 阿拉坦仓, 张鸿庆, 钟万牺. 矩阵多元多项式的带余除法及其应用. 应用数学和力学, 2000, 21: 661-668

5 Vainberg M M. Variational Methods for the Study of Nonlinear Operators. San Francisco: Holden-Day, 1964

6 钟万㭷. 弹性力学求解新体系. 大连: 大连理工大学出版社, 1995

7 Yao W A, Zhong W X, Lim C W. Symplectic Elasticity. Singapore: World Scientific Publishing, 2009

8 Lim C W, Lü C F, Xiang Y, Yao W A. On new symplectic elasticity approach for exact free vibration solutions of rectangular Kirchhoff plates. Internat J Engrg Sci, 2009, 47: 131-140

9 Zhong Y, Li R, Liu Y M, Tian B. On new symplectic approach for exact bending solutions of moderately thick rectangular plates with two opposite edges simply supported. Internat J Solids Structures, 2009, 46: 2506-2513

10 Xu X S, Zhang W X, Li X, et al. An application of the symplectic system in two-dimensional viscoelasticity. Internat J Engrg Sci, 2006, 44: 897-914

11 Christiane T. Spectral theory of block operator matrices and applications. London: Imperial College Press, 2008

12 Hou G L, Alatancang. On the feasibility of variable separation method based on Hamiltonian system for plane magnetoelectroelastic solids Chinese Physics B, 2008, 27: 2753-2758

13 侯国林, 阿拉坦仓. 对边简支的矩形平面弹性问题的辛本征展开定理. 应用数学和力学, 2010, 31: 1181-1190

14 陈晓敏, 侯国林, 程婷, 等. 矩形中厚板 Hamilton 方程的解析解. 固体力学学报, 2011, 32: 611-618

15 吴德玉, 阿拉坦仓. 无穷维 Hamilton 算子特征函数系的 Cauchy 主值意义下的完备性. 中国科学 A 辑, 2008, 38: 904-912

16 Hou G L, Alatancang. Completeness of eigenfunction systems for off-diagonal infinite-dimensional Hamiltonian operators. Commun Theor Phys (Beijing), 2009, 51: 200-204

17 Lim C W, Cui S, Yao W A. On new symplectic elasticity approach for exact bending solutions of rectangular thin plates with two opposite sides simply supported. Internat J Solids Structures, 2007, 44: 5396-5411

18 黄俊杰, 阿拉坦仓, 范小英. 无穷维 Hamilton 算子的谱结构. 中国科学 A 辑, 2008, 38: 71-78

19 Hou G L, Alatancang. Spectra of off-diagonal infinite-dimensional Hamiltonian operators and their applications to plane elasticity problems. Commun Theor Phys (Beijing), 2009, 51: 200-204

20 Elias M S, Rami S. Fourier Analysis: An Introduction. Princeton: Princeton University Press, 2003

\section{Completeness of generalized eigenfunction systems of infinite- dimensional Hamiltonian operators and its application in Elas- ticity}

\section{HOU Guolin \& Alatancang}

Abstract By using the structural characteristics of the infinite-dimensional Hamiltonian operators, a necessary and sufficient condition is given for generalized eigenfunction systems to be complete in the sense of Cauchy principal value. The above function systems consist of the eigenfunction and Jordan eigenfunction of the operators. Moreover, the result is applied to plate bending problems. The related conclusion offers a theoretical guarantee for the separation of variables in Hamiltonian system (the new systematic methodology for theory of elasticity).

Keywords Hamiltonian system, infinite-dimensional Hamiltonian operator, completeness, Cauchy principal value, plate bending equation

MSC(2010) 47A75, 74B10, 47A45

doi: 10.1360/012011-348 